\title{
Transrectal ultrasound-guided prostate biopsy: periprostatic block versus caudal block for analgesia-a randomized trial
}

\author{
Oluwatobi Ayodeji Fasola ${ }^{1 *} \mathbb{D}$, Augustine Oghenewyin Takure ${ }^{1,2}$ and Olayiwola B. Shittu,2
}

\begin{abstract}
Background: Transrectal ultrasound (TRUS)-guided prostate biopsy is a potentially painful procedure, due to the insertion of the TRUS probe in the anus and multiple passes of the biopsy needle through the rectum and prostate. Several methods of reducing pain and discomfort have been described. These include intra-rectal local anaesthetic gel (IRLA) instillation, periprostatic nerve block (PPNB), caudal block (CB) and oral analgesics. CB has potential complications of dural puncture and anaesthetic failure, while PPNB may be complicated by intravascular injection with systemic local anaesthetic toxicity. Only few studies have compared transrectal PPNB with CB with equivocal results. This study compared transrectal PPNB to CB in terms of efficacy of analgesia and incidence of complications.

Methods: A prospective randomized clinical trial was carried out among 80 consenting patients with an indication for TRUS-guided prostate biopsy in the Urology division of [BLINDED FOR PEER REVIEW]. Eighty participants were each randomized to either of Group A (CB with $10 \mathrm{ml}$ of 2\% lidocaine) or Group B (PPNB with a total of $20 \mathrm{ml}$ of 1\% lidocaine). Pain was assessed using an 11-point numerical rating scale (NRS), and questions on satisfaction with the procedure and willingness for a repeat procedure were asked. The incidence of complications was also recorded.

Results: There were no significant differences in the mean ages, body mass indices (BMls), prostate-specific antigen (PSA) levels, digital rectal examination (DRE) findings and prostate sizes between the two groups. The mean NRS scores at administration of block, insertion of TRUS probe, prostate biopsy, $30 \mathrm{~min}$ and 1 day after biopsy were $2.9 \pm 2.3,2.1 \pm 2.2,3.1 \pm 2.6,1.4 \pm 2.2$ and $0.2 \pm 0.4$ respectively for $C B$ and $3.1 \pm 2.2,2.3 \pm 1.2,2.8 \pm 2.7,1.4 \pm 1.7$ and $0.3 \pm 0.5$, respectively, for the PPNB group. There were no significant differences between the mean scores in both groups. There were also no statistically significant differences in the incidences of complications in both groups.
\end{abstract}

Conclusion: The two methods of analgesia are similar in efficacy and are equally safe to employ in the performance of TRUS-guided prostate biopsy. Both methods can be learned to increase the repertoire of the urologist when faced with a TRUS-guided prostate biopsy.

Trial registration: PACTR, PACTR202012779661309. Registered 11th December 2020-Retrospectively registered, https://pactr.samrc.ac.za/TrialDisplay.aspx?TrialID=14564.

Keywords: Analgesia, Caudal analgesia, Transrectal ultrasound, Prostate biopsy, Periprostatic nerve block

*Correspondence: fasola.oluwatobi@yahoo.com

${ }^{1}$ Department of Surgery, University College Hospital, Ibadan, Nigeria

Full list of author information is available at the end of the article

\section{Background}

Prostate cancer is the second most common malignancy in men, second only to skin cancer [1]. The diagnosis of prostate cancer is suspected in patients with elevated serum levels of prostate-specific antigen (PSA) and abnormal digital rectal examination (DRE) 
findings [2]. To confirm the diagnosis of prostate cancer, a histological diagnosis is required. The current preferred method for obtaining prostate tissue for histology is by transrectal ultrasound (TRUS)-guided biopsy of the prostate [3]. This involves the use of a transrectal ultrasound probe to guide a core biopsy needle to obtain prostate tissue via the rectum. The needle, loaded on a biopsy gun, advances through the rectal mucosa and prostate gland to retrieve prostatic tissue.

The procedure of transrectal prostate biopsy may cause considerable pain due to the passage of the biopsy needle through the rectal mucosa and prostate capsule. In the early days, many urologists employed no form of analgesia for prostate biopsies. They believed that the rectum and prostate gland were not sensitive to the passage of the biopsy needle $[4,5]$. However, some men reported considerable painful experiences with the procedure. There are reports of up to two-thirds of patients experiencing pain with no form of analgesia [6, 7]. Different local or regional anaesthetic methods have been employed to prevent pain arising from the biopsy. These include caudal block (CB), periprostatic nerve block (PPNB), intraprostatic injection of local anaesthetic (IPLA) or the instillation of intra-rectal local anaesthetic gel (IRLA). PPNB involves injecting a local anaesthetic around the neurovascular bundles as they reach the prostate at the junction of the seminal vesicles and prostate gland, while caudal block, also known as caudal analgesia, involves injecting local anaesthetic into the epidural space of the sacral spinal canal through the sacral hiatus.

In Nigeria, at the time of this writing, more centres perform digitally guided prostate biopsies and the prevalent methods of analgesia being CB and IRLA [8]. The number of centres with the capacity for TRUS-guided prostate biopsy has, however, begun to increase across the country. Thus, it is increasingly possible to provide transrectal ultrasound-guided PPNB. This study compared the analgesic effect of PPNB to the currently widely practiced CB for TRUS-guided prostate biopsy as assessed by pain scores on the numerical rating scale (NRS) during and after the procedure.

Specific objectives:

1. To determine whether PPNB is better or worse than CB in achieving analgesia for TRUS-guided prostate biopsy. Primary outcome is to find a statistically significant difference in the mean numerical rating scale pain scores between the two groups.

2. To determine and compare the incidence of complications of PPNB and CB for analgesia.

\section{Methods}

The study was a randomized clinical trial. It was carried out at the [BLINDED FOR PEER REVIEW] among male patients attending the clinics of the urological surgery division. It was a parallel trial design with a 1:1 allocation ratio. A total of 80 patients were enrolled, with 40 subjects in each arm. A simple randomization technique was employed in the assignment of patients. This was achieved with a randomization schedule which was generated using an online computer software. Patients currently or recently on anticoagulants or antiplatelet medications or those with bleeding disorders, acute prostatitis, and painful anal conditions were excluded.

\subsection{Sample size determination}

The sample size was estimated with parameters to achieve a significant clinically acceptable margin of 1 point with a standard deviation of 1.5 on the NRS as seen in a previous study. [9] The power of the study was set at $80 \%$ and at a level of statistical significance of 0.05:

$$
N=2 \times\left(\frac{z_{1-\alpha}+z_{1-\beta}}{\delta_{0}}\right)^{2} \times s^{2}
$$

where $N=$ sample size of one arm;

$Z_{1-\alpha}($ standard normal deviate $)=1.96$;

$Z_{1-\beta}=0.845$

$\delta_{0}$ (a clinically acceptable difference $)=1$;

$S^{2}$ (pooled standard deviation $)=1.5$

$N=2 \times[(1.96+0.845) / 1]^{2} \times 1.5^{2}=35.4=$ approximately 36 patients per arm.

A total of 80 patients were enrolled, with 40 subjects in each arm. Eight extra patients were enrolled to give allowance for a $10 \%$ drop-out rate.

\subsection{Protocol for caudal block}

For every patient assigned to the caudal block group, $160 \mathrm{mg}$ of intravenous gentamicin was administered as pre-procedure antibiotic prophylaxis. Thereafter, the patient was positioned prone and the skin of the lower back was prepared with standard skin preparation solution, and then, $10 \mathrm{ml}$ of $2 \%$ lidocaine was injected with a $22 \mathrm{G}$ needle into the epidural space through the sacral hiatus. Inadvertent injection into a blood vessel was prevented by aspirating prior to injection and repositioning the needle if blood was aspirated into the syringe. The adequacy of the block was then assessed with a perineal needle prick and finding a lax anal sphincter when a rectal examination was done. Occasionally, a mild elongation of the penis was also 
observed. The patient was then repositioned in the left lateral decubitus position, and after a 5-min wait, the prostate biopsy was commenced.

\subsection{Protocol for periprostatic nerve block}

Each patient was placed in the left lateral position, and following the administration of intravenous antibiotic (gentamicin $160 \mathrm{mg}$ ) prophylaxis, the transrectal ultrasound probe was inserted into the rectum. Under TRUS guidance, with a $22 \mathrm{G} 20$-cm-long spinal needle, $5 \mathrm{ml}$ of $1 \%$ lidocaine was infiltrated into the neurovascular bundle around the junction of the prostate and seminal vesicles bilaterally and on both sides of the prostatic apex to make a total of $20 \mathrm{ml}$ of $1 \%$ lidocaine infiltrated. Appropriate and adequate injection was confirmed by observing a hypoechoic wheal on the ultrasound screen as the anaesthetic agent was injected. Inadvertent intravascular injection was prevented by test aspiration prior to infiltration and adjusting the needle if blood was aspirated. After a 5-min wait, the prostate biopsy was commenced.

\subsection{Prostate biopsy protocol}

Following a digital rectal examination, a $5.0-9.0 \mathrm{MHz}$ transrectal ultrasound probe (SonoScape Medical Corp., Shenzhen, China) was inserted into the rectum, covered with a condom sheath and lubricated by ultrasound gel (Guang Dong University of Technology, China). The volume and echogenicity of the prostate gland were then assessed on the ultrasound machine. Following this, a twelve-core systematic prostate biopsy protocol was performed using a reusable biopsy gun with an 18G core biopsy needle (Geotek Medical, Osb-Ankara, Turkey). At the end of the procedure, digital pressure was placed, with a gauze pack, on the prostate via the rectum to control bleeding.

\subsection{Pain assessment protocol}

The pain scores of the participants were assessed with the 11-point numerical rating scale (NRS) at five different moments during the procedure: $\mathrm{T} 1-$ when the caudal block or periprostatic block was administered; T2-when the TRUS probe was inserted; T3-during the prostate biopsy; T4-thirty minutes after the biopsy; and T5-the day after the biopsy. (The participants were contacted on phone.) The participants were educated on how to use the NRS, they all had a good understanding of the ordinal properties of the numbers and only had to verbally score their perception of pain with the NRS at the different moments pain was assessed.

\subsection{Data analysis}

The data collected were analysed with the IBM SPSS Statistics for Windows, Version 20.0 (IBM Corp., Armonk,
N.Y., USA). Parametric and nonparametric tests of significance (Chi-square, $t$ test and Mann-Whitney $\mathrm{U}$ test) were used in evaluating the differences between the groups. Statistical significance was defined for the study as $p<0.05$.

\subsection{Expertise}

Urologists and trainees at the study centre have routinely performed $\mathrm{CB}$ for procedures like prostate biopsy, rigid cystoscopy, urethral dilation. It is a routine part of training program. All the CBs were performed by the first author. The first author acquired extra training for PPNB and became proficient over 2 years when the TRUS machine was introduced to their urological practice. He performed all the PPNBs in this study.

\section{Results}

\subsection{Recruitment of participants}

During the study period, which was carried out from May 2018 to December 2018, a total of 80 subjects were recruited, and 40 men were allocated randomly into each arm of the trial. The flow diagram for the recruitment is as follows (Fig. 1).

\subsection{Group characteristics in terms of age, body mass index (BMI), prostate-specific antigen (PSA), prostate size, DRE and TRUS findings and previous caudal block or prostate biopsy}

There were no statistically significant differences in the mean age, body mass index, pre-biopsy prostate-specific antigen level, prostate size of the patients, DRE and TRUS findings in either group. There was also no significant difference in the proportion of men in both groups who had prior biopsy and caudal block (Table 1).

\subsection{Comparison of pain scores between groups}

Table 2 shows the comparison of the mean pain scores between the two different groups. The mean pain score was highest at the moment of prostate biopsies for the $\mathrm{CB}$ group (3.1 \pm 2.6$)$, while it was highest at the moment of administering block for the PPNB group (3.1 \pm 2.2$)$. The difference in mean pain score was greatest at the moment of taking the prostate biopsies, with the men in the $\mathrm{CB}$ group experiencing a mean pain score that was $10 \%$ greater than the mean score of the PPNB group. However, this difference in mean pain score was not statistically significant. There was no statistically significant difference in the pain scores at any other moment of the procedure.

There was a slightly higher mean pain score at insertion of the TRUS probe in the PPNB group compared to the $\mathrm{CB}$ group, and this was, however, not statistically significant. Figure 2 further shows that at the insertion 


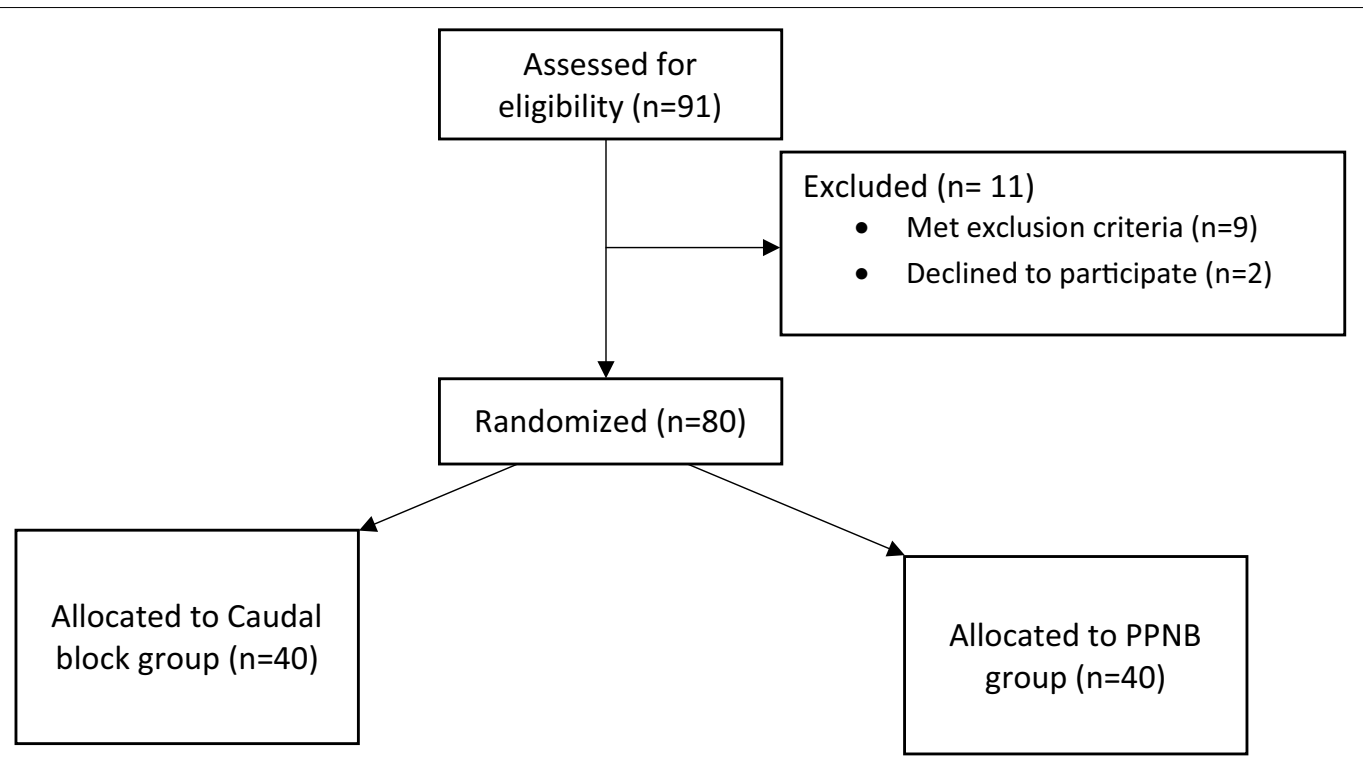

Fig. 1 Flow diagram showing how participants were recruited and allocated to the two intervention groups

Table 1 Comparison of mean ages, BMI, PSA, TRUS prostate volumes, prior procedures and TRUS prostate findings between the group A and group B

\begin{tabular}{|c|c|c|c|}
\hline & Group A (CB) & Group B (PPNB) & $p$ value \\
\hline & Mean \pm SD & Mean \pm SD & \\
\hline Age (years) & $69.2 \pm 8.7$ & $68.5 \pm 6.4$ & 0.67 \\
\hline \multirow[t]{2}{*}{ BMI $\left(\mathrm{kg} / \mathrm{m}^{2}\right)$} & $24.9 \pm 4.7$ & $27.0 \pm 5.1$ & 0.06 \\
\hline & Median (IQR) & Median (IQR) & \\
\hline PSA (ng/ml) & $28.1(16.8-98.4)$ & $50.5(17.2-211.9)$ & 0.40 \\
\hline \multirow[t]{2}{*}{ TRUS prostate size $\left(\mathrm{cm}^{3}\right)$} & $60.2(38.3-94.0)$ & $57.8(36.5-95.0)$ & 0.98 \\
\hline & Number (\%) & Number (\%) & \\
\hline \multicolumn{4}{|l|}{ Prior prostate biopsy? } \\
\hline Yes & $8(20)$ & $6(15)$ & 0.56 \\
\hline No & $32(80)$ & $34(85)$ & \\
\hline \multicolumn{4}{|l|}{ Prior caudal block? } \\
\hline Yes & $8(20)$ & $7(17.5)$ & 0.73 \\
\hline No & $32(80)$ & $33(82.5)$ & \\
\hline \multicolumn{4}{|l|}{ TRUS echogenicity? } \\
\hline Homogeneous & $13(27.5)$ & $11(32.5)$ & 0.63 \\
\hline Heterogeneous & $27(72.5)$ & $29(67.5)$ & \\
\hline
\end{tabular}

$C B$ caudal block, PPNB periprostatic nerve block, BMI body mass index, SD standard deviation, PSA prostate-specific antigen, TRUS transrectal ultrasound

of the TRUS probe, the pain scores for men in the PPNB group only ranged from 0 to 4 , while they ranged from 0 to 9 in the CB group. Also, the modal score was 0 for the CB group (30\%), while it was 3 for the PPNB group (32.5\%).

\subsection{Comparison of satisfaction with procedure} and willingness to repeat biopsy procedure and incidence of complications between groups

A higher proportion of the patients who had PPNB (72.5\%) than those who had CB (65\%) were satisfied with 
Table 2 Modal pain score at administration of block was 3 for both groups, while at insertion of probe, the modal scores were 0 and 3 for CB and PPNB, respectively

\begin{tabular}{lllll}
\hline & $\begin{array}{l}\text { Group A (CB) } \\
\text { Mean } \pm \text { SD }\end{array}$ & $\begin{array}{l}\text { Group B (PPNB) } \\
\text { Mean } \pm \text { SD }\end{array}$ & Mean difference \\
\hline At administration of block & $2.9 \pm 2.3$ & $3.1 \pm 2.2$ & -0.2 & 0.56 \\
At insertion of TRUS probe & $2.1 \pm 2.2$ & $2.3 \pm 1.2$ & -0.2 & 0.18 \\
At taking prostate biopsy & $3.1 \pm 2.6$ & $2.8 \pm 2.7$ & 0.3 & 0.40 \\
30-min post-procedure & $1.4 \pm 2.2$ & $1.4 \pm 1.7$ & 0.0 & 0.48 \\
1-day post-procedure & $0.2 \pm 0.4$ & $0.3 \pm 0.5$ & -0.1 & 0.32 \\
\hline
\end{tabular}

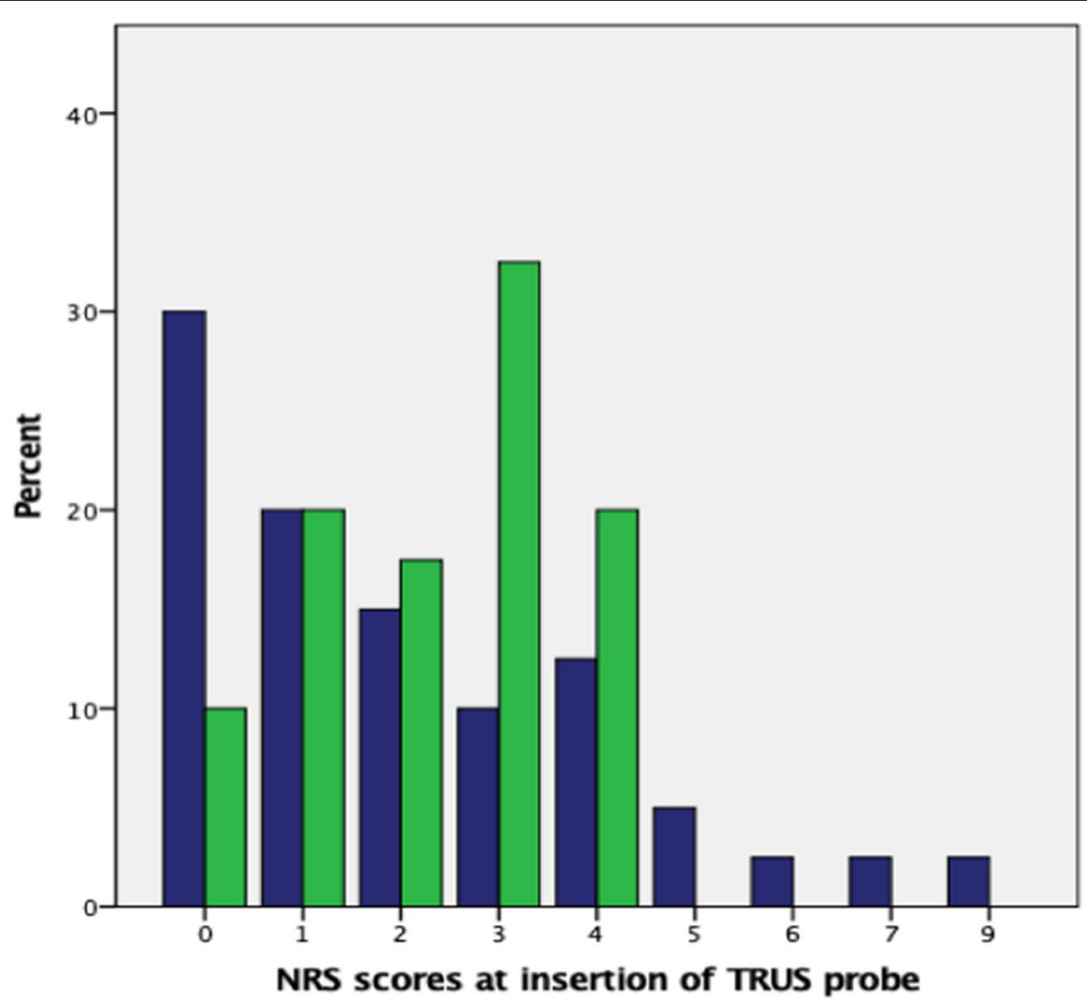

Intervention group $(\mathrm{A}=\mathrm{CB} ; \mathrm{B}=\mathrm{PPNB})$

$\square$ B

Fig. 2 Bar chart showing the percentages of men per reported NRS pain score for each intervention group at the insertion of the TRUS probe. CB (Group A); PPNB (Group B)

the method of block used. This was, however, not statistically significant. Furthermore, a similar proportion $(42.5 \%)$ of men were willing to repeat the prostate biopsy procedure using the same method for blocking pain in both intervention groups. There was a low rate of complications in all the participants, with no significant difference between the groups (Table 3).

\section{Discussion}

\subsection{Group characteristics}

Transrectal ultrasound-guided prostate biopsy is an established method for obtaining prostate tissue for histological diagnosis. Prevention and/or alleviation of a painful experience is important for the procedure. The results showed no significant differences between patients in the two arms of the study in terms of their mean age, BMI, PSA levels and previous medications/ procedures. The findings were similar to the study carried out by Horinaga and colleagues [9] in Japan in which the average ages of the patients were not statistically different between the PPNB and CB groups and were close to the average ages in this study. Furthermore, in the study by Urabe et al. [10] the average BMI values were 24.1 \pm 3.0 and $23.9 \pm 2.9$ in the PPNB and CB group, respectively, 
Table 3 Comparison of the proportion of patients who were satisfied with and who were willing to have repeat prostate biopsy using the same method of analgesia between the two groups, and the incidence of complications following prostate biopsy between groups

\begin{tabular}{|c|c|c|c|}
\hline & $\begin{array}{l}\text { Group A (CB) } n=40 \\
\text { Number }(\%)\end{array}$ & $\begin{array}{l}\text { Group B } \\
\text { (PPNB) } n=40 \\
\text { Number }(\%)\end{array}$ & $p$ value \\
\hline \multicolumn{4}{|c|}{ Satisfaction with procedure } \\
\hline Satisfied & $26(65 \%)$ & $29(72.5 \%)$ & \multirow[t]{3}{*}{0.77} \\
\hline Indifferent & $9(22.5 \%)$ & $7(17.5 \%)$ & \\
\hline Not satisfied & $5(12.5 \%)$ & $4(10 \%)$ & \\
\hline \multicolumn{4}{|c|}{ Willingness to repeat biopsy with similar block } \\
\hline Willing & $17(42.5 \%)$ & $17(42.5 \%)$ & \multirow[t]{3}{*}{0.96} \\
\hline Indifferent & $13(32.5 \%)$ & $14(35 \%)$ & \\
\hline Not willing & $10(25 \%)$ & $9(22.5 \%)$ & \\
\hline \multicolumn{4}{|l|}{ Hematuria? } \\
\hline Yes & $6(15 \%)$ & $6(15 \%)$ & \multirow[t]{2}{*}{0.96} \\
\hline No & $34(85 \%)$ & $34(85 \%)$ & \\
\hline \multicolumn{4}{|c|}{ Febrile reaction? } \\
\hline Yes & $1(2.5 \%)$ & $1(2.5 \%)$ & \multirow[t]{2}{*}{1.00} \\
\hline No & $39(97.5)$ & $39(97.5 \%)$ & \\
\hline \multicolumn{4}{|c|}{ Urinary retention? } \\
\hline Yes & $2(5 \%)$ & $0(0 \%)$ & \multirow[t]{2}{*}{0.15} \\
\hline No & $38(95 \%)$ & $40(100 \%)$ & \\
\hline
\end{tabular}

and the difference was not statistically significant, similar to findings in our study. The PSA levels and prostate volumes, however, were higher in our study than in the study by Horinaga et al. [9] which compared anaesthetic efficacy between PPNB and CB in 100 men (50 per arm). In their study, the mean PSA levels were $8.5 \pm 10.4 \mathrm{ng} / \mathrm{ml}$ and $10.0 \pm 7.8 \mathrm{ng} / \mathrm{ml}$ in the PPNB and CB groups, respectively. This could be explained by the fact that our participants are Africans with a racial predilection for larger prostates and PSA levels [11].

\subsection{Comparison of pain scores between groups}

Our study showed that patients reported a higher pain score at insertion of the TRUS probe in the PPNB group compared to the CB group, though this was not statistically significant. Similarly, Urabe et al. [10] reported a statistically significant higher average pain score at the point of probe insertion for PPNB + IRLA group compared to the CB+IRLA group. This is probably due to the fact that insertion of the TRUS probe precedes the administration of the PPNB in the PPNB group, while the administration of $\mathrm{CB}$ preceded probe insertion which also had the effect of relaxing the anal sphincter. This point may confer an advantage on CB over PPNB as analgesia for prostate biopsy.

There was no significant difference in the experience of pain at administration of block in our study. However, Urabe et al. [10] reported a significantly higher average pain score of $2.9 \pm 2.1$ in the CB + IRLA group than in the PPNB+IRLA group $(2.0 \pm 1.9)$ at the moment of administering the analgesia. This could have been as a result of the fact that in their study, IRLA was instilled prior to the administration of PPNB.

The modal pain scores were 0 for both $\mathrm{CB}$ and PPNB groups, and there was no statistical difference in the mean pain scores for both groups in our study. This implied no advantage of either method of analgesia over the other in regard to the analgesic effect at prostate biopsy. Urabe et al. [10] observed similar findings, with no significant difference in the pain scores between the PPNB+IRLA and CB+IRLA groups. However, Horinaga et al. [9] in Japan found that the PPNB group had a significantly lower average pain score of $1.1 \mathrm{com}-$ pared to 2.1 in the caudal block group $(p=0.01)$. They suggested that their dosage of $10 \mathrm{ml}$ of $1 \%$ lidocaine for the $\mathrm{CB}$ group was probably inadequate considering the anatomic capacity of the sacral epidural space. Also, they submitted that the prostate presumably has other innervation from preaortic and sacrococcygeal nerve groups. In a Nigerian study by Obi et al. [12] of 75 men (25 randomized to three groups - no analgesia; PPNB; and saddle block), there was a significantly higher pain score in the periprostatic lidocaine injection group $(4.6 \pm 2.3)$ than in the CB group $(0.7 \pm 1.6)$. However, in their study, the periprostatic injection of lidocaine was digitally guided, and therefore, the anaesthetic agent may not have been delivered precisely at the plane between the prostate and seminal vesicles. It is important to note that the dosages and regimens of the local anaesthetic agents used in these studies differ, and this may have accounted for the lack of consensus in the results. A summary of the protocols and pain scores of the reviewed literature is presented in Table 4.

The sensation of pain after the prostate biopsy as shown by our results was not statistically different between the groups. With either method of analgesia, post-procedural pain was minimal. This was similar to the findings of $\mathrm{Na}$ Wang and colleagues [13] which showed no significant difference in the pain scores at 30-min post-procedure; the mean scores were $0.9 \pm 1.1$ and $1.1 \pm 1.1$ for $\mathrm{CB}$ and PPNB + IRLA, respectively $(p=0.27)$. The pain scores a day following prostate biopsy in our study were similar to the findings of the study by Horinaga et al. [9] a day after prostate biopsies. 
Table 4 Protocols, findings and conclusions of studies that compared CB with PPNB

\begin{tabular}{|c|c|c|c|c|c|c|c|}
\hline \multirow[t]{2}{*}{ Study } & \multicolumn{2}{|l|}{ Protocol } & \multirow[t]{2}{*}{ Number of patients } & \multicolumn{2}{|c|}{$\begin{array}{l}\text { Pain score at prostate } \\
\text { biopsy }\end{array}$} & \multirow[t]{2}{*}{$p$ value } & \multirow[t]{2}{*}{ Conclusion } \\
\hline & A & B & & A & B & & \\
\hline Na Wang et al. [13] & $\mathrm{CB}^{\mathrm{a}}$ & $P P N B^{\beta}+I R L A^{\delta}$ & 190 (95 each) & $1.4 \pm 1.3$ & $1.9 \pm 1.6$ & 0.01 & CB better \\
\hline Horinaga et al. [9] & $\mathrm{CB}^{\varphi}$ & $\mathrm{PPNB}^{\varphi}$ & 100 (50 each) & $2.1 \pm 1.9$ & $1.1 \pm 1.3$ & 0.01 & PPNB better \\
\hline Urabe et al. [10] & $C B^{\varphi}+I R L A^{\varepsilon}$ & $\operatorname{PPNB}^{\varphi}+\mathrm{IRLA}^{\varepsilon}$ & 532 (266 each) & $3.0 \pm 2.6$ & $2.9 \pm 2.5$ & 0.56 & No difference \\
\hline Obi et al. [12] & $\mathrm{CB}^{\lambda}$ (saddle) & $\operatorname{PPNB}^{\varphi}$ (digit guided) & $\begin{array}{l}75 \text { (25 each-added a } 3^{\text {rd }} \\
\text { group with no analgesia) }\end{array}$ & $0.7 \pm 1.6$ & $4.6 \pm 2.3$ & 0.00 & CB better \\
\hline Our study & $\mathrm{CB}^{\mu}$ & $\mathrm{PPNB}^{\sigma}$ & 80 (40 each) & $3.1 \pm 2.6$ & $2.8 \pm 2.7$ & 0.40 & No difference \\
\hline
\end{tabular}

Anaesthetic agents used: ${ }^{\alpha} 20 \mathrm{ml} 1.2 \%$ lidocaine; ${ }^{\beta} 10 \mathrm{ml} 1 \%$ lidocaine $+0.5 \%$ ropivacaine; ${ }^{\delta} 0.3 \%$ oxybuprocaine gel; ${ }^{\varphi} 10 \mathrm{ml} 1 \%$ lidocaine; ${ }^{\varepsilon} 10 \mathrm{ml} 1 \%$ lidocaine gel;

${ }^{\lambda} 0.5 \mathrm{ml} 0.5 \%$ bupivacaine; ${ }^{\mu} 10 \mathrm{ml} 2 \%$ lidocaine; and ${ }^{\sigma} 20 \mathrm{ml} 1 \%$ lidocaine

\subsection{Comparison of satisfaction with procedure and willingness to repeat biopsy procedure and incidence of complications between groups}

The results of this study revealed that there was no statistical difference in the level of satisfaction experienced by the participants of either groups. This was in contrast to the report by $\mathrm{Na}$ Wang and colleagues [13]. In their study of 190 men, over half (46.7\%) in the CB group while only $24.2 \%$ of the men in PPNB + IRLA group reported 'excellent' satisfaction with the procedure $(p=0.014)$. However, the difference in proportion was less pronounced when the sum of men who reported either 'excellent' or 'good' levels of satisfaction were compared between the groups: $84.7 \%$ versus $75.8 \%$ for CB and PPNB + IRLA, respectively.

The willingness to repeat the prostate biopsy using the same method of analgesia was similar across the groups. Obi et al. [12] reported similar proportions of men willing to have a repeat biopsy; $72 \%$ of the men who had PPNB and $88 \%$ men who had saddle block (CB) were willing to have a repeat prostate biopsy $(p=0.55)$.

The results showed a low incidence of complications, with no significant difference between the groups. This was similar to the findings by Na Wang et al. [13] where out of 95 persons per group, one man, 2 men and 6 men had hematuria, fever and urinary retention, respectively in the CB group, while 3 men, 4 men and 4 men with similar complications, respectively, in the PPNB + IRLA group. Similarly, there was no significant difference in the incidence of complications between the groups in the study by Urabe et al. [10].

\subsection{Limitations of the study}

This study did not account for the average number of prostate biopsy cores obtained or number of passes of the biopsy needle in the two groups. The study also did not explore the need for oral analgesia (or the use of self-medicated analgesics) in the days following the prostate biopsy as this may have been a pointer to the sensation of post-procedural pain. However, most of the patients reported very low pain scores at 1 day after the procedure and this probably would not have affected the findings and conclusions of this study.

\section{Conclusion}

The results of this study revealed that PPNB and CB are equally effective in achieving analgesia for TRUS-guided prostate biopsies. CB seemed to have an advantage of providing a lax anal sphincter and reducing pain during TRUS probe insertion. Similar proportions of patients in both groups were satisfied and were willing to have a repeat biopsy. Finally, the incidence of complications of either method was low and there was no statistical difference between the groups.

\section{Recommendations}

Though not shown by the results of this study, PPNB may have an advantage in situations where $\mathrm{CB}$ might be difficult or impossible such as in obese patients or those with a fused sacral hiatus. It also requires no change in the position of the patient. Urologists should acquire the skills to perform both $\mathrm{CB}$ and PPNB, to increase the armamentarium of methods of analgesia for TRUSguided prostate biopsies. Such that in cases when the equally effective caudal block fails or is not feasible, the urologist may offer an alternative in PPNB or vice versa.

\section{CONSORT reporting guidelines}

The study adheres to CONSORT guidelines, and a completed CONSORT checklist has been attached to the manuscript.

\section{Abbreviations}

BMI: Body mass index; CB: Caudal block; DRE: Digital rectal examination; IPLA: Intra-prostatic local anaesthetic injection; IRLA: Intra-rectal local anaesthetic instillation; LA: Local anaesthetic; MHz: Megahertz; NRS: Numerical rating scale; 
PPNB: Periprostatic nerve block; PSA: Prostate-specific antigen; TRUS: Transrectal ultrasound; VAS: Visual analogue scale; $\times 2$ : Chi-square.

\section{Supplementary Information}

The online version contains supplementary material available at https://doi. org/10.1186/s12301-021-00185-3.

Additional file 1. CONSORT checklist for study.

\section{Acknowledgements}

Not applicable.

\section{Authors' contributions}

OAF performed most of the PPNB, CB and the prostate biopsies. He also carried out the data analysis and contributed to the manuscript. AOT performed some of the prostate biopsies and contributed to the writing of the manuscript. OBS contributed to writing the manuscript. All authors read and approved the final manuscript.

\section{Funding}

None.

\section{Availability of data and materials}

The datasets used and/or analysed during the current study are available from the corresponding author on reasonable request.

\section{Declarations}

\section{Ethics approval and consent to participate}

Informed written consent was obtained from each patient enrolled in the study. Ethical approval was obtained from the University of Ibadan/University College Hospital Institution review board. IRB Number UI/EC/17/0453.

\section{Consent for publication}

Not applicable.

\section{Competing interests}

The authors declare that they have no competing interests.

\section{Author details}

'Department of Surgery, University College Hospital, Ibadan, Nigeria. ${ }^{2}$ Department of Surgery, College of Medicine, University of Ibadan, Ibadan, Nigeria.

Received: 29 December 2020 Accepted: 12 June 2021

Published online: 29 June 2021

\section{References}

1. Menegoz F, Lutz JM, Mousseau M, Orfeuvre H, Schaerer R (2019) Descriptive epidemiology of prostate cancer. World J Oncol 10(2):63-89

2. Daniyal M, Siddiqui ZA, Akram M, Asif HM, Sultana S, Khan A (2014) Epidemiology, etiology, diagnosis and treatment of prostate cancer. Asian Pac J Cancer Prev 15(22):9575-9578

3. Ramey JR, Halpern EJ, Gomella LG (2007). In: Wein A, Kavoussi L, Novick A, Partin A, Peters C (eds) Ultrasonography and biopsy of the prostate, 9th edn. Saunders, Philadelphia, pp 2883-2895

4. Amling CL, Spencer CR, Roberts JL (2004) Practice patterns in transrectal ultrasound guided prostate biopsy: results of a questionnaire survey of practicing urologists. J Urol 171:1809

5. Crundwell M, Cooke P, Wallace D (1999) Patients' tolerance of transrectal ultrasound-guided prostatic biopsy: an audit of 104 cases. BJU Int 83:792-795

6. Collins GN, Lloyd SN, Hehir M, McKelvie GB (1993) Multiple transrectal ultrasound-guided prostatic biopsies-true morbidity and patient acceptance. Br J Urol 71(4):460-463

7. Seymour H, Perry MJA, Lee-Elliot C, Dundas D, Patel U (2001) Pain after transrectal ultrasonography-guided prostate biopsy: the advantages of periprostatic local anaesthesia. BJU Int 88(6):540-544

8. Ikuerowo SO, Popoola AA, Olapade-Olaopa EO, Okeke LI, Shittu OB, Adebayo SA et al (2010) Caudal block anesthesia for transrectal prostate biopsy. Int Urol Nephrol 42(1):19-22

9. Horinaga M, Nakashima J, Nakanoma T (2006) Efficacy compared between caudal block and periprostatic local anesthesia for transrectal ultrasound-guided prostate needle biopsy. Urology 68(2):348-351

10. Urabe F, Kimura T, Shimomura T, Onuma H, Yamamoto T, Sasaki H et al (2017) Prospective comparison of the efficacy of caudal versus periprostatic nerve block, both with intrarectal local anesthesia, during transrectal ultrasonography-guided prostatic needle biopsy. Scand J Urol 51(4):245-250

11. Udeh El, Ozoemena OFN, Ogwuche E (2019) The relationship between prostate volume and international prostate symptom score in Africans with benign prostatic hyperplasia. Niger J Med 21(3):290-295

12. Obi AO, Okafor VU, Nnodi PI (2011) Prospective randomized trial of spinal saddle block versus periprostatic lignocaine for anesthesia during transrectal prostate biopsy. Urology 77(2):280-285

13. Wang N, Fu Y, Ma H, Wang J, Gao Y (2016) Advantages of caudal block over intrarectal local anesthesia plus periprostatic nerve block for transrectal ultrasound guided prostate biopsy. Pak J Med Sci 32(4):978-982

\section{Publisher's Note}

Springer Nature remains neutral with regard to jurisdictional claims in published maps and institutional affiliations.

\section{Submit your manuscript to a SpringerOpen ${ }^{\circ}$ journal and benefit from:}

- Convenient online submission

- Rigorous peer review

- Open access: articles freely available online

- High visibility within the field

- Retaining the copyright to your article

Submit your next manuscript at $\boldsymbol{\nabla}$ springeropen.com 